\title{
Métabolisme protéique de l'agneau nouveau-né. IV. Conséquences de l'ingestion d'acides aminés et de lactose
}

\author{
P Patureau Mirand 1*, ML Houlier 1, J Prugnaud 1, \\ M Dalle 2, J Grizard 1 \\ avec la collaboration technique de M Sallas, A Selle \\ 1 INRA Theix, Laboratoire d'étude du métabolisme azoté, 63122 Ceyrat, France; \\ 2 Université Blaise Pascal, Clermont-Ferrand II, \\ Laboratoire de physiologie animale, 63180 Aubière, France
}

(Reçu le 24 août 1989; accepté le 4 janvier 1990)

\begin{abstract}
Résumé - Les conséquences sur le métabolisme protéique de l'ingestion d'acides aminés seuls ou avec du lactose ont été étudiées chez l'agneau nouveau-né. Huit agneaux ont été séparés de leur mère immédiatement après leur naissance et nourris toutes les heures, soit avec une solution d'acides aminés et de peptides obtenus par hydrolyse ménagée de protéines de lactosérum (4 agneaux; régime AP), soit avec la même solution additionnée de lactose (4 agneaux; régime APL). Dès $2 \mathrm{~h} 30 \mathrm{~min}$ après leur naissance, ils ont été soumis à une perfusion de $L-\left[4,5-{ }^{3} \mathrm{H}\right]$ leucine dans une veine jugulaire pendant $6 \mathrm{~h}$, pour étudier les flux de leucine et apprécier l'intensité de la synthèse protéique dans différents tissus ou organes.

Les teneurs plasmatiques en glucose et en insuline augmentaient après la naissance chez les agneaux recevant le régime APL ef diminuaient chez ceux recevant l'autre aliment (fig 1). Les teneurs en cortisol plasmatique n'étaient pas significativement différentes. Les teneurs en acides aminés libres plasmatiques ne variaient pas après $4,5 \mathrm{~h}$ et dépendaient de la quantité ingérée. En outre, les concentrations en thréonine, valine, isoleucine, leucine, tyrosine et lysine étaient plus faibles chez les agneaux APL que chez ceux recevant l'aliment AP (fig 2). Le flux plasmatique de leucine correspondant au catabolisme des protéines corporelles était significativement plus faible chez les agneaux APL, dans la mesure où, avec les 2 régimes, le flux plasmatique de leucine alimentaire correspondait à la quantité de leucine ingérée. Les taux de synthèse protéique n'étaient pas significativement différents dans le foie, la peau, le muscle, le poumon, le cerveau et le corps entier (tableau II). Dans la peau, le muscle et le corps entier, ces taux étaient voisins de ceux mesurés précédemment chez des agneaux nourris avec du colostrum. L'augmentation de la masse des protéines corporelles consécutive à l'ingestion simultanée de lactose et d'acides aminés semble résulter d'une réduction du catabolisme des protéines qui pourrait être induite par la réponse insulinique observée avec le régime APL.
\end{abstract}

agneau / nouveau-né / métabolisme protéique / acide aminé / lactose

Summary - Protein metabolism in newborn lambs. IV. Effect of amino acid feeding. A study was made on protein metabolism and hormonal changes following birth in nowborn lambs fed amino acids alone or in combination with lactose. Eight newborn lambs taken from their mother immediately after birth were fed hourly for $8 \mathrm{~h}$, either with a solution of peptides and free amino acids obtained

* Corespondance et tirés à part 
by mild hydrolysis of whey proteins (4 lambs; diet AP) or with the same solution + lactose (4 lambs; $\operatorname{diet} A P L) . L-\left[4,5^{3} H\right]$ leucine was continuously perfused into a jugular vein for $6 h$ when the lambs were 2 h 30 min old. Plasma glucose and insulin levels increased after birth in APL lambs whereas they decreased in the AP; these differences were significantly different (fig 1). Plasma cortisol levels remained unchanged throughout the experiment. Free essential amino acid levels did not vary when lambs were older than $4.5 \mathrm{~h}$; they depended on the corresponding amino acid intakes.

Plasma free threonine, valine, isoleucine, leucine, tyrosine and lysine were lower in APL than in $A P$ lambs (fig 2). The plasma leucine irreversible loss and leucine oxidation were higher in AP than in $A P L$ lambs (table 1). The plasma flux of leucine from whole body protein breakdown was lower in APL than in AP lambs inasmuch as the plasma flux of dietary leucine may be estimated by the amounts of leucine ingested in both cases. No significant difference was found for the fractional synthesis rates of tissue proteins such as liver, skin, skeletal muscle, lung, brain and whole body (table II). These rates for skin, muscle and whole body were close to those previously measured in colostrum fed lambs. The increase in whole body protein accretion resulting from lactose feeding in combination with amino acids seemed to result from a decreased protein breakdown that could be mediated by the insulin response.

lamb / newborn / protein metabolism / amino acid / lactose

\section{INTRODUCTION}

L'ingestion de colostrum par l'agneau nouveau-né s'accompagne d'une stimulation de la protéosynthèse musculaire qui est associée à un accroissement du flux plasmatique de leucine et du catabolisme de cet acide aminé (Patureau Mirand et al, 1985a). Pour rechercher les causes de ces phénomènes, l'étude des conséquences de l'ingestion de divers nutriments majeurs du colostrum (lactose, acides aminés) sur le métabolisme protéique de l'agneau nouveau-né a été entreprise. Dans une première expérience, il a été montré que l'ingestion de lactose seul stimule la protéosynthèse musculaire, réduit le catabolisme de la leucine, et ne modifie pas son flux plasmatique (Patureau Mirand et al, 1986). Un effet des acides aminés ingérés avec le colostrum peut aussi être envisagé puisque, chez l'homme, le flux plasmatique de la leucine et son catabolisme oxydatif varient en fonction des quantités ingérées (Meguid et al, 1986). De plus, in vivo, chez le rat en croissance, l'activité de la protéosynthèse musculaire dépend étroitement de l'apport protéique (Jepson et al, 1988). Toutefois, selon Preedy et Garlick (1986), la perfusion d'acides aminés ne stimule la protéosynthèse dans le muscle du rat en croissance à jeun que lorsque du glucose est ajouté à la perfusion grâce à l'action de l'insuline, dont les effets seraient accentués par la perfusion d'acides aminés (Garlick et Grant, 1988).

Dans ce travail donc, les conséquences sur le métabolisme protéique de l'agneau nouveau-né de l'ingestion d'acides aminés seuls ou en association avec du lactose seront comparées à celles de l'ingestion de colostrum ou de lactose seul. Cela permettra de préciser le rôle de certains nutriments colostraux et de poursuivre l'analyse de l'effet de l'ingestion de lactose sur le métabolisme protéique d'agneaux nouveau-nés en étudiant les effets de la consommation simultanée d'acides aminés.

\section{MATÉRIEL ET MÉTHODES}

\section{Animaux et alimentation}

Huit agneaux mâles de race limousine ou croisés lle de France $x$ Romanov $x$ Limousin, pe- 
sant entre 2,9 et $4,1 \mathrm{~kg}$, sont séparés de leur mère dès leur naissance. Ils sont installés chacun dans une cage placée dans un local ventilé ayant une température de $21^{\circ} \mathrm{C}$ et une hygrométrie voisine de $70 \%$. Un cathéter est alors introduit sous anesthésie locale dans chaque veine jugulaire externe. Dès $1 \mathrm{~h}$ après leur naissance, ces 8 agneaux, répartis en 2 lots, sont alimentés toutes les heures au biberon. Les uns (agneaux AP) reçoivent à chaque repas $50 \mathrm{ml}$ d'une solution de $\mathrm{NaCl} 0,9 \%$ additionnée d'un mélange d'acides aminés et de peptides (40 $\mathrm{g} / \mathrm{l}$ ); les autres (agneaux APL) sont nourris avec la même solution à laquelle $35 \mathrm{~g}$ de lactose ont été ajoutés. Le mélange peptidique utilisé provient d'une hydrolyse ménagée de protéines du lactosérum de vache; il contient surtout des peptides de moins de 11 résidus (plus de 60\% dont moins de $8 \%$ sous forme d'acides aminés libres - Sopharga, 14480 Creuilly, France).

\section{Perfusion ef prélèvements}

Deux heures et demie après leur naissance, les agneaux sont soumis à une perfusion continue de $L-[4,5-3 H]$ leucine (activité spécifique 37 $\mathrm{GBq} / \mathrm{mmol}$; Amersham), pendant $6,5 \mathrm{~h}$ a raison de $12 \mathrm{MBq} / \mathrm{h}$. Des prélèvements de sang jugulaire sont effectués $2 h, 4 h$ et $6 h$ après le début de la perfusion pour la détermination des teneurs en acides aminés libres, en glucose, en insuline et en cortisol du plasma, ainsi que pour celle de la radioactivité spécifique de la leucine libre. Un prélèvement sanguin est aussi réalisé à l'áge de 1 h 30 (une demi-heure après le premier repas) pour la mesure de la glycémie. En fin de perfusion, les agneaux sont sacrifies par égorgement après anesthésie générale au pentobarbital sodique. Le foie, le muscle long dorsal, le cerveau, les poumons sont rapidement prélevés et congelés dans l'azote liquide avec le reste du corps.

\section{Analyses et calculs}

Les teneurs plasmatiques en glucose sont déterminées par la méthode à la glucose-oxydase; celles de l'insuline par radioimmunologie au moyen de la trousse CEA-SORIN-INSIK1; celles du cortisol par radiocompétition (Dalle et Delost,
1976). Les méthodes utilisées pour déterminer la radioactivité de la leucine libre ou protéique dans les tissus et celles pour mesurer les concentrations en acides aminés libres du plasma et des tissus ont été décrites précédemment (Patureau Mirand et al, 1985a, b). Les méthodes de calcul du flux plasmatique de leucine (c'est-à-dire la perte irréversible), des flux de catabolisme de la leucine, de son utilisation pour la protéosynthèse et des quantités libérées par la protéolyse sont fondées sur celles préconisées par Waterlow et al (1978); elles ont été détaillées précédemment (Patureau Mirand et al, 1985a; Patureau Mirand et al, 1986). Une estimation de l'intensité du taux de renouvellement des protéines (Ks exprimé en \%/j) dans les différents tissus ou organes a été effectuée selon la méthode décrite par Garlick et al (1973). En bref, elle se déduit de l'évolution en fonction du temps du rapport de la radioactivité spécifique de la leucine incorporée dans les protéines (Sb) à celle de la leucine dans le compartiment précurseur pour la synthèse protéique (S) qui est decrite par la relation :

$$
\frac{\mathrm{Sb}}{\mathrm{s}}=\frac{\lambda}{\lambda-\mathrm{Ks}} \times \frac{1-\mathrm{e}^{-\mathrm{Ks} \times t}}{1-\mathrm{e}^{-\lambda \times \mathrm{t}}}-\frac{\mathrm{Ks}}{\lambda-\mathrm{Ks}}
$$

$\lambda$ est une constante caractéristique de l'évolution de la radioactivité spécifique de la leucine dans le compartiment précurseur de la synthèse protéique (exprimée en $\mathrm{j}^{-1}$ ); les valeurs qui lui ont été affectées, sont celles préconisées par Waterlow et al.(1978). t est la durée de la perfusion (en jours).

Deux estimateurs du taux de renouvellement des protéines dans chacun des tissus ou organes étudiés peuvent être calculés par itération : Ksi si la valeur de $\mathrm{S}$ est assimilée a celle de la radioactivité spécifique de la leucine libre tissulaire (valeurs présentées) ou Ksp si elle est assimilee à celle de la leucine libre plasmatique (voir en Discussion les raisons du choix de la présentation des Ksi).

\section{Statistiques}

Le degré de liaison entre variables est apprécié à l'aide du coefficient de corrélation de Spearman. Les moyennes entre lots sont comparées 
à l'aide du test $t$ de Student lorsque les variables ne sont pas étroitement dépendantes d'une autre variable comme la quantité d'acides aminés ingérée. Quand elles le sont, leurs moyennes sont comparées à l'aide d'une analyse de covariance (Snedecor et Cochran, 1971).

\section{RÉSULTATS}

\section{Teneurs en glucose, insuline et cortisol plasmatiques}

Les teneurs en glucose plasmatique (fig 1) augmentent dans les 2 lots jusque vers $4,5 \mathrm{~h}$; cet accroissement se poursuit jusqu'à $8,5 \mathrm{~h}$ chez les agneaux APL alors qu'une diminution est observée chez les agneaux AP à 6,5 et $8,5 \mathrm{~h}$. II en résulte des différences de glycémie entre les 2 lots, significatives à ces temps $(P<0,05)$. Les concentrations en insuline (fig 1) varient peu jusqu'à $6,5 \mathrm{~h}$ dans le plasma des agneaux des 2 lots; elles augmentent fortement dans le plasma des agneaux APL à $8,5 \mathrm{~h}$ et la différence avec celles des agneaux AP devient significative $(P<$ $0,05)$. La corrélation entre les concentrations en ces 2 composés est significative ( $r=0,65$ pour 24 observations). Les teneurs en cortisol varient peu entre 4,5 et $8,5 \mathrm{~h}$ (83 et $85 \mathrm{ng} / \mathrm{ml}$ respectivement) et ne sont pas significativement différentes dans les lots AP et APL ( $81 \pm 12$ et $88 \pm 9$ $\mathrm{ng} / \mathrm{ml}$ respectivement).

\section{Teneurs plasmatiques en acides aminés libres}

Les teneurs en chacun des acides aminés indispensables libres et tyrosine ne varient pas significativement au cours de l'expérience. Les résultats présentés (fig 2) correspondent à la teneur moyenne des 3 observations réalisées sur chaque agneau aux âges de 4,5-6,5 et 8,5 h. Dans la plupart des cas, ces teneurs sont corrélées avec les quantités ingérées. Les coefficients de corrélation de Spearman sont les suivants : thréonine 0,60 ; valine 0,79 ; méthionine 0,90 ; isoleucine 0,79 ; leucine 0,79 ; tyrosine 0,87 ; phénylalanine 0,81 ; lysine 0,76 ; histidine 0,81 ; arginine 0,14 . Du fait de cette liaison entre les teneurs plasmatiques et les quantités ingérées, l'effet régime a été étudié sur les moyennes ajustées par covariance. Les teneurs de ces composés sont plus faibles dans le plasma des agneaux APL que dans celui des agneaux de l'autre lot. Ces différences sont significatives $(P<0,05)$ pour la thréo-
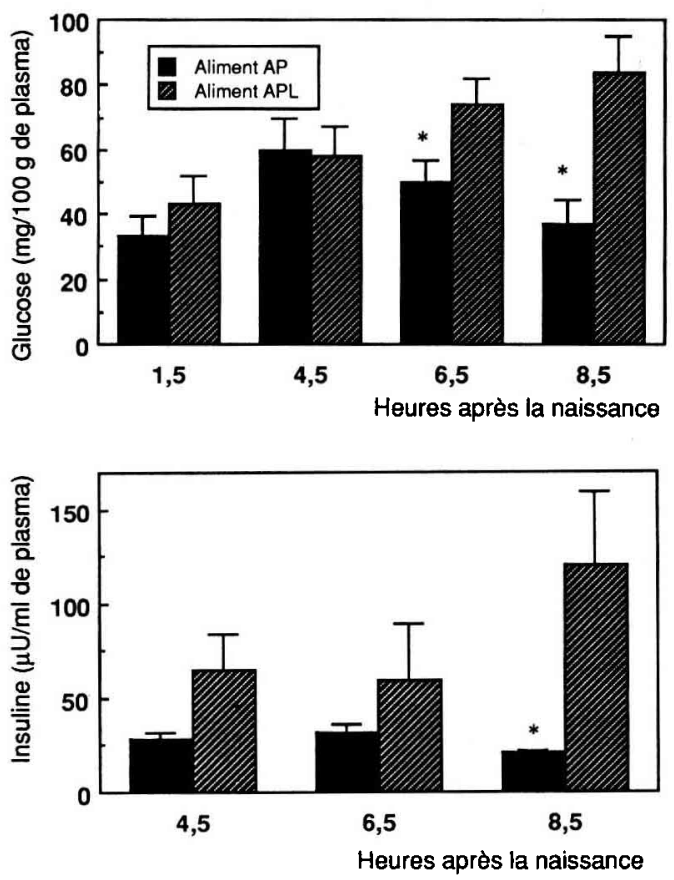

Fig 1. Evolution des teneurs plasmatiques en glucose et en insuline (moyenne et écart type) après la naissance chez des agneaux nouveaunés nourris avec les aliments AP et APL. " Différence significative entre les 2 lots $(P<0,05)$. 

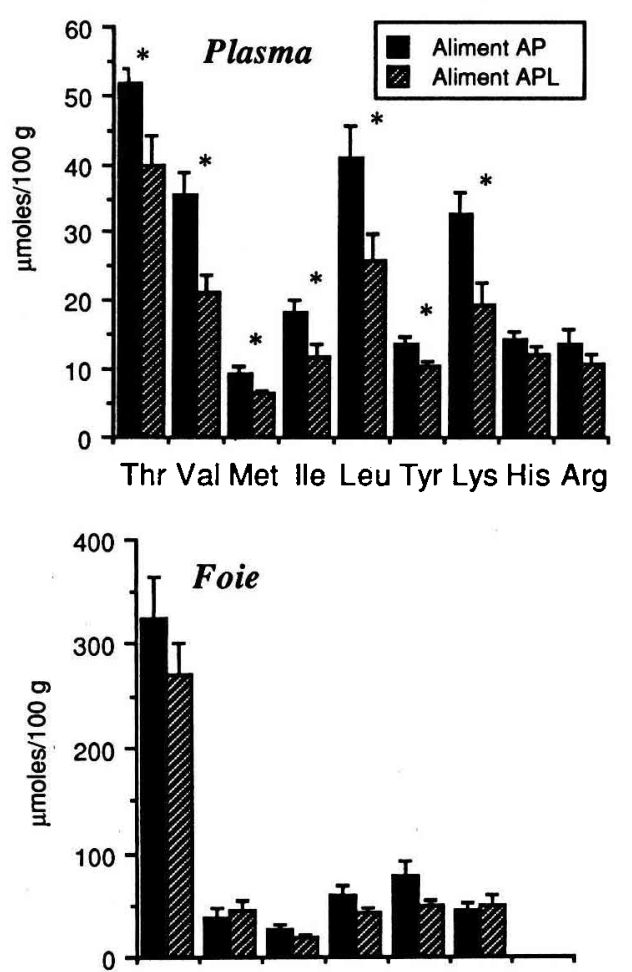

Thr Val lle Leu Lys His Arg
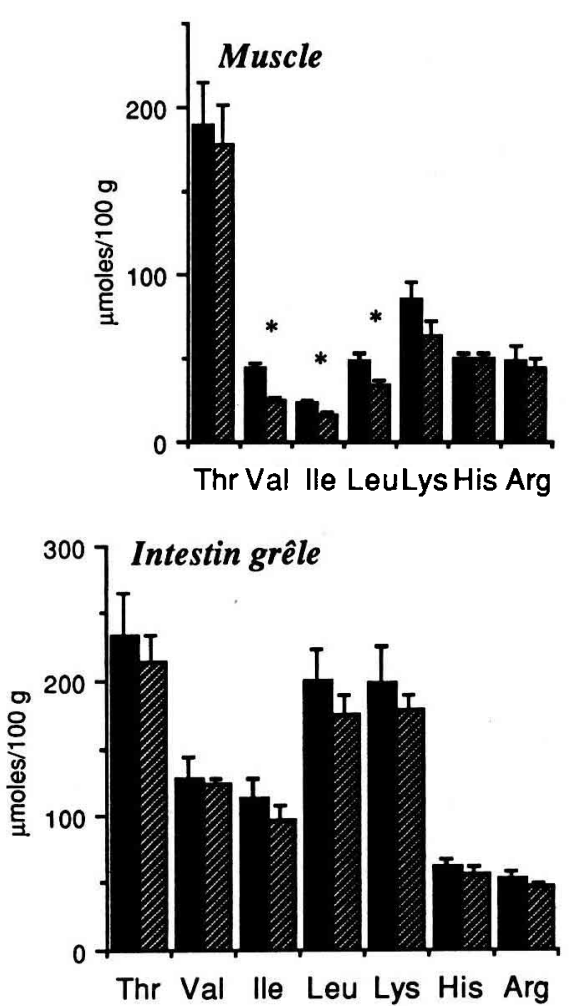

Fig 2. Teneurs en acides aminés libres du plasma, du muscle long dorsal, du foie et de l'intestin grêle (moyenne et écart type). * Différence significative entre les 2 lots $(P<0,05)$.

nine, la valine, la méthionine, l'isoleucine, la leucine, la tyrosine et la lysine.

Les teneurs en acides aminés non indispensables libres n'évoluent pas significativement au cours de l'expérience à l'exception de celles en glycine qui diminuent significativement entre 4,5 et $8,5 \mathrm{~h}$ de 70,0 à $49,4 \mu$ moles $/ 100 \mathrm{ml}$, dans les 2 lots. Les teneurs en acides aminés non indispensables libres du plasma sont plus faibles, bien que de façon non significative, chez les agneaux APL que chez les agneaux AP. II n'existe cependant pas de corrélation significative entre les teneurs plasmati- ques en glucose et en acides aminés libres non indispensables.

\section{Teneurs en acides aminés libres des tissus}

Les teneurs en acides aminés libres du foie et de l'intestin grêle ne sont pas différentes dans les 2 lots d'agneaux, exception faite de la glycine qui est en concentration plus faible dans le foie des agneaux APL que dans celui des agneaux AP; ces concentrations en acides aminés ne sont 
pas significativement corrélées avec les quantités ingérées. En revanche, dans le muscle, les teneurs en valine, isoleucine, leucine et lysine libres sont significativement corrélées avec les quantités ingérées; de plus, elles sont significativement plus faibles, lysine exceptée, chez les agneaux APL que chez les agneaux AP.

\section{Flux plasmatiques de leucine (tableau I)}

La radioactivité spécifique de la leucine libre plasmatique atteint les valeurs observées au plateau $2 \mathrm{~h}$ après le début de la perfusion puisque, dès ce temps-là, elle est égale à $104 \%$ de la valeur moyenne au plateau. Les flux plasmatiques de leucine dépendant de la quantité de leucine ingérée $(r=0,80)$, les comparaisons entre les valeurs mesurées dans les 2 lots ont été effectuées par analyse de covariance en utilisant la quantité de leucine ingérée comme covariable. La perte irréversible de leucine plasmatique est significativement plus élevée chez les agneaux qui consomment l'aliment AP ne contenant que la solution peptidique que chez ceux qui sont nourris avec celui contenant le mélange de peptides et de lactose (APL). Les flux plasmatiques de leucine utilisée pour la synthèse protéique sont du même ordre de grandeur selon qu'ils sont calculés par différence entre la perte irréversible de leucine plasmatique et le flux de leucine cata-

Tableau I. Flux plasmatique de la leucine et ses composantes (moyenne \pm écart type).

Valeurs en $\mu$ moles $\cdot \mathrm{h}^{-1} \cdot \mathrm{kg}^{-1}$. (1) assimilé à la quantité de leucine ingérée; (2) avec le flux de leucine alimentaire comme covariable; (3) calculé selon la procédure décrite dans Matériel et Méthodes; (4) différence entre le flux plasmatique et le flux de leucine catabolisée; (5) différence entre le flux plasmatique et le flux de leucine alimentaire; (6) différence entre le flux de leucine utilisée pour la protéosynthèse et le flux de leucine libérée par la protéolyse. " $P<0,05$; ${ }^{* *} P<0,01$.

$\begin{array}{llll}\text { Régimes } & A P & A P L & P\end{array}$

Nombre d'agneaux

Flux de leucine alimentaire (1)

Flux plasmatique de leucine

- mesuré

- ajusté par covariance (2)

Flux de catabolisme de la leucine (3)

- mesuré

- ajusté par covariance (2)

Flux de leucine utilisée pour

la protéosynthèse (4)

Flux de leucine libérée par

la protéolyse (5)

Flux de leucine fixée (6)

\section{4}

$434 \pm 33$

$645 \pm 27$

$660 \pm 9$

$280 \pm 27$

$298 \pm 7$

$367 \pm 5$

$211 \pm 11$

$156 \pm 11$
$355 \pm 6$

\section{4}

$452 \pm 25$

$599 \pm 20$

$583 \pm 13$

$246 \pm 22$

$227 \pm 10$

$147 \pm 22$

$208 \pm 17$

$P$

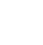


bolisée ou à partir des taux de renouvellement des protéines tissulaires en supposant que la radioactivité spécifique de la leucine dans le compartiment précurseur de la protéosynthèse est égale à celle de la leucine libre plasmatique. Ces flux sont voisins dans les 2 lots (367 et 355 $\left.\mu \mathrm{mol} \cdot \mathrm{h}^{-1} \cdot \mathrm{kg}^{-1}\right)$. En revanche, le flux de catabolisme de la leucine est plus élevé chez les agneaux AP que chez les agneaux APL; il en est de même du flux de leucine libérée par la protéolyse. II en résulte que la différence entre la quantité de leucine plasmatique utilisée par la protéosynthèse et celle libérée par la protéolyse, quantité qui correspond à celle de leucine retenue dans les protéines tissulaires, est plus faible chez les agneaux AP que chez ceux qui consomment la solution peptidique additionnée de lactose. II n'y a pas de liaison statistique significative entre chacun de ces paramètres et les teneurs plasmatiques en insuline ou en cortisol, sauf en ce qui concerne le flux de leucine libérée par la protéolyse et les teneurs en insuline ( $r=$ $-0,70$ ).

\section{Taux de renouvellement des protéines tissulaires (tableau II)}

Les taux de renouvellement des protéines tissulaires (Ksi), calculés en supposant que la radioactivité spécifique de la leucine dans le compartiment précurseur pour la protéosynthèse est égale à celle de la leucine libre intratissulaire, sont rapportés dans le tableau II. Ces taux sont les plus élevés dans le foie et les poumons et les plus faibles dans le muscle long dorsal. Ils ne sont pas significativement différents dans les 2 lots bien que l'écart entre les mesures effectuées dans la peau soit relativement important.

\section{DISCUSSION}

\section{Digestion des peptides des aliments}

L'utilisation d'un mélange peptidique au lieu d'un mélange d'acides aminés a été

Tableau II. Taux de renouvellement des protéines tissulaires (Ksi, moyenne \pm écart type $\% \cdot{ }^{-1}$ ).

\begin{tabular}{lcc}
\hline Régimes & $A P$ & $A P L$ \\
\hline Nombre d'agneaux & 4 & 4 \\
Foie & $42,5 \pm 2,0$ & $48,7 \pm 4,3$ \\
Peau & $30,0 \pm 3,9$ & $21,2 \pm 1,7$ \\
Long dorsal & $14,3 \pm 2,1$ & $12,8 \pm 0,4$ \\
Poumon & $48,2 \pm 5,9$ & $48,6 \pm 3,7$ \\
Cerveau & $34,8 \pm 4,3$ & $34,1 \pm 3,1$ \\
Corps entier & $21,0 \pm 1,0$ & $19,3 \pm 0,4$ \\
\hline
\end{tabular}


retenue, car l'absorption d'acides aminés paraît plus rapide sous cette forme (Gray et Cooper, 1971; Matthews, 1983; Silk et al, 1985). Cela a été confirmé récemment chez le porc par Rérat et al (1988) avec des mélanges peptidiques issus de l'hydrolyse enzymatique de protéines de lait et comparables à celui utilisé dans ce travail. Les quantités de peptides à distribuer avaient été calculées à partir des estimations des quantités d'acides aminés indispensables entrées dans le sang sous forme libre après ingestion de colostrum (Patureau Mirand et al, 1985b), de façon à en apporter par l'aliment une quantité légèrement supérieure (+ $18 \%$ en moyenne). Les teneurs plasmatiques en acides aminés indispensables libres observées sont en accord avec celles mesurées après ingestion de colostrum, compte tenu des différences entre les quantités ingérées dans cet essai et les estimations des quantités fournies par le colostrum. Ainsi, ces teneurs sont plus élevées pour la thréonine, la valine, l'isoleucine, la leucine et la lysine qui ont été ingérées en quantités plus grandes que les quantités fournies par le colostrum; celles en tyrosine, phénylalanine, histidine et arginine sont du même ordre de grandeur ou inférieures, comme c'est le cas des apports alimentaires.

L'étroite liaison observée entre les quantités ingérées et les teneurs sanguines semble indiquer que l'apport d'acides aminés sous forme de peptides a été efficace. II semblerait avoir été aussi efficace que la fourniture d'acides aminés par des protéines de lait à des agneaux âgés d'une semaine. En effet, l'application des relations établies dans ce cas (Patureau Mirand et al, 1985b) pour calculer les quantités d'acides aminés apportées à l'organisme des agneaux nouveau-nés de l'expérience présente, fournit des estimations très proches des quantités ingérées (quantités fournies à l'organisme $=0,95 \times$ quantités ingérées $+20, r=0,90$ ) pour les 9 acides aminés indispensables étudiés et la tyrosine. Ceci peut être rapproché des résultats de Rérat et al (1988) qui indiquent que les quantités d'acides aminés entrées dans le sang de la veine porte, au cours des 2 heures qui suivent la perfusion duodénale d'une solution peptidique à des porcs, correspondent sensiblement aux quantités perfusées, dans le cas de la plus faible dose.

\section{Variations des teneurs plasmatiques en glucose et insuline}

La glycémie des agneaux du lot AP est légèrement plus élevée que celle d'agneaux du même âge restés à jeun et placés dans les mêmes conditions (Patureau Mirand et al, 1985b, 1986). Celle des agneaux nourris avec le mélange peptidique associé au lactose, qui est 2 fois plus élevée, reste inférieure à celle d'agneaux recevant une solution ne contenant que du lactose : 176 $\mathrm{mg}$ pour $100 \mathrm{~g}$ (Patureau Mirand et al, 1986). Cette différence peut s'expliquer par la différence des quantités de lactose consommées $(1,75$ et $2,25 \mathrm{~g} / \mathrm{h}$ respectivement dans les 2 études), ainsi que par la réponse insulinique. En effet, l'insulinémie des agneaux nourris avec le mélange peptidique est 3 fois plus élevée que celle des agneaux qui consomment l'aliment ne contenant que du lactose; elle est voisine de celle observée dans le plasma d'agneaux de même âge nourris au colostrum de vache $(81 \mu \mathrm{U} / \mathrm{ml})$. L'insulinémie des agneaux AP est relativement basse et proche de celle mesurée après ingestion de lactose seul; elle est légèrement supérieure à celle des agneaux à jeun de cette expérience-là. II semble donc que chez l'agneau nouveau-né, l'ingestion de peptides, comme celle de lactose accroît faj- 
blement l'insulinémie; un tel effet a déjà été décrit chez d'autres espèces, récemment chez le rat (Preedy et Garlick, 1986) ou le porc (Rérat et al, 1988). Le mélange de lactose et de peptides renforce l'effet de ces 2 composés chez l'agneau nouveauné, comme chez le rat, le porc ou l'homme. Cela permet de penser que la sécrétion d'insuline peut être stimulée par ces nutriments dès les premières heures de la vie d'une façon peut-être un peu plus efficace que semblaient l'indiquer les travaux de Philipps et al (1981).

\section{Adaptation du métabolisme protéique à l'ingestion d'une solution peptidique additionnée ou non de lactose}

\section{Signification physiologique des paramètres d'appréciation des aspects dynamiques du métabolisme protéique}

L'analyse de l'effet de nutriments comme le lactose et des peptides sur le métabolisme protéique, grâce à l'étude des flux plasmatiques de la leucine, suppose qu'ils peuvent être estimés avec précision et sont représentatifs des phénomènes étudiés. Or s'il est relativement aisé de déterminer la perte irréversible de leucine libre plasmatique, ce critère n'est qu'une estimation partielle de l'intensité du métabolisme de l'ensemble des acides aminés comme a pu le montrer Obled (1987), chez le rat, à l'aide de perfusions simultanées de 5 acides aminés indispensables. De plus, les estimations des quantités de leucine plasmatique utilisée pour la protéosynthèse, par les voies du catabolisme ou libérées par la protéolyse ne peuvent être réellement considérées comme des mesures précises des quantités de leucine mises en jeu, mais plutôt comme des descripteurs univoques de ces phénomènes. II en est de même de l'estimation des taux de renouvellement des protéines tissulaires. Les taux, Ksp ou Ksi, obtenus en considérant soit la radioactivité spécifique de la leucine plasmatique, soit celle de la leucine intratissulaire comme représentative de celle de la leucine du pool précurseur de la protéosynthèse, sont très différents. La comparaison de ces résultats avec ceux obtenus par la méthode de référence, la méthode de la surcharge de valine (Attaix et al, 1986), indique que les meilleures estimations de ces taux de renouvellement correspondent sensiblement à la moyenne arithmétique des Ksi et Ksp pour des tissus ou organes comme le muscle, le poumon et le cerveau ainsi que le corps entier (Patureau Mirand, en préparation). Toutefois, l'effet des traitements étudiés sur ces paramètres est le même quel que soit le mode de calcul utilisé.

\section{Effet de l'ingestion de la solution peptidique}

L'ingestion du régime AP produit un flux plasmatique de leucine élevé, légèrement plus élevé que celui mesuré après ingestion de colostrum $\left(541 \mu \mathrm{mol} \bullet \mathrm{h}^{-1} \cdot \mathrm{kg}^{-1}\right.$ selon Patureau Mirand et al, 1985a). Cette différence (104 $\left.\mu \mathrm{mol} \cdot \mathrm{h}^{-1} \cdot \mathrm{kg}^{-1}\right)$ correspond en grande partie à celle des flux de leucine alimentaire $\left(88 \mu\right.$ moles $\left.\bullet^{-1} \cdot \mathrm{kg}^{-1}\right)$. Cela suggère que la quantité de leucine libérée par la protéolyse est peu différente chez les agneaux AP et chez ceux recevant le colostrum. Le catabolisme accru de la leucine $(+51 \%)$ par rapport à celui mesuré chez les agneaux nourris au colostrum correspond à l'accroissement des teneurs plasmatiques en leucine. La compilation de nos résultats chez l'agneau nouveau-né indique qu'il existe une étroite relation linéaire entre les teneurs plasmatiques et le flux de catabolisme de la leucine $(r=0,95)$, comme cela a déjà été décrit notamment 
chez le rat (Harper et Benjamin, 1984) ou l'homme (Meguid et al, 1986). Le flux de leucine plasmatique libérée par la protéolyse est comparable chez les agneaux AP et ceux nourris au colostrum. Ces valeurs sont voisines de celles mesurées chez des agneaux de même âge restés à jeun (180 $\mu$ moles $\cdot \mathrm{h}^{-1} \cdot \mathrm{kg}^{-1}$ en moyenne).

\section{Conséquences du supplément de lactose}

L'ingestion du supplément de lactose s'est accompagnée d'une réduction du flux plasmatique de leucine. Une légère diminution de l'utilisation de la leucine plasmatique pour la protéosynthèse et surtout pour le catabolisme, associée à une nette réduction de l'entrée dans le compartiment plasmatique de leucine provenant de la protéolyse tissulaire en sont les principales causes. De tels effets ont été décrits chez le mouton de 9 mois (Nissen et Ostaszewski, 1985) et chez le porc en croissance (Reeds et al, 1987), lorsque les animaux reçoivent un supplément d'énergie. Les réductions des teneurs en acides aminés libres du plasma et dans une moindre mesure du muscle peuvent ainsi s'expliquer par la diminution de la protéolyse chez les agneaux nourris avec l'aliment APL. Cette réduction des teneurs en acides aminés libres circulants explique alors que le catabolisme de la leucine soit moins intense chez ces agneaux que chez ceux qui reçoivent la solution peptidique seule. Cet effet est comparable à celui décrit lors de la comparaison des flux de leucine chez des agneaux à jeun ou recevant une solution de lactose (Patureau Mirand et al, 1986). En revanche, dans les tissus, l'ingestion du supplément de lactose n'a pas eu d'effet significatif sur la protéosynthèse, contrairement à ce qui avait été constaté dans le travail cité précédemment. Cela peut provenir du fait que la pro- téosynthèse était déjà élevée chez les agneaux qui consommaient l'aliment AP. Au contraire, au niveau du corps entier, l'ingestion de lactose paraît avoir réduit légèrement l'utilisation de la leucine pour la protéosynthèse.

II est difficile de discerner les facteurs qui expliquent l'ensemble des phénomènes observés. Si certains résultats rappellent ceux de Horn et al (1983) chez le fœetus ovin soumis à des perfusions de glucose, d'acides aminés ou d'insuline, seuls ou associés, d'autres en diffèrent. Certes l'action de l'insuline peut être envisagée dans la mesure où certains de ses effets sur le métabolisme protéique ont été constatés : réduction de la protéolyse et du catabolisme oxydatif des acides aminés. $\mathrm{Ce}$ pendant, l'activité de la protéosynthèse musculaire n'a pas été accrue chez les agneaux du lot APL au-delà de ce qu'elle était chez les agneaux du lot AP, alors que l'insulinémie est de 2 à 6 fois plus élevée. L'ingestion de nutriments (lactose, peptides, lactose et peptides) stimule, de façon voisine dans ces 3 cas, la protéosynthèse musculaire (Ksi $=13,9 ; 14,3 ; 12,8 \% / \mathrm{j}$ lorsque les agneaux sont nourris respectivement avec une solution de lactose, les aliments $A P$ et $A P L$ ) par rapport à ce qui est observé chez des agneaux nouveaunés restés à jeun pendant $8 \mathrm{~h}$ (Ksi $=7,2$ $\% / j)$. Ceci se produit alors que les augmentations de l'insulinémie sont faibles avec le lactose ou les peptides seuls et importantes avec le mélange lactose et peptides. L'effet stimulant de l'insuline sur la protéosynthèse pourrait être plus efficace pour les valeurs basses comme cela a été montré chez le rat (Millward et al, 1983), l'effet inhibiteur de la protéolyse étant plus marqué avec des insulinémies élevées. II se pourrait aussi que la méthode de perfusion pour mesurer l'intensité de la protéosynthèse n'ait pas permis de déceler une éventuelle augmentation tardive de la pro- 
téosynthèse, correspondant aux valeurs les plus élevées de l'insulinémie chez les agneaux du lot APL. L'action d'autres tacteurs $\left(I G F_{1}\right.$ et hormones thyroïdiennes, notamment) peut être envisagée; celle du cortisol n'a pu être mise en évidence, peutêtre parce que la réactivité surrénalienne est difficile à stimuler après les épreuves de la naissance, durant le premier jour.

\section{CONCLUSION}

L'utilisation d'une solution peptidique, associée ou non à du lactose, a permis de réaliser un apport contrôlé d'acides aminés à l'organisme d'agneaux nouveau-nés. Cet apport a provoqué une réaction du métabolisme protéique comparable à l'ingestion de colostrum en ce qui concerne les flux plasmatiques de leucine (flux total, flux pour la protéosynthèse, pour le catabolisme) et la protéosynthèse musculaire. L'addition de lactose a eu des effets qui ont modulé les conséquences de l'ingestion de la solution peptidique : les flux plasmatiques ont été moins accrus et surtout le flux plasmatique de leucine libérée par la protéolyse a été réduit. En revanche, il n'y a pas additivité des effets sur la protéosynthèse musculaire de l'ingestion de lactose et d'acides aminés.

Certains des effets décrits suggèrent que l'action des nutriments (acides aminés, glucose) sur le métabolisme protéique d'agneaux nouveau-nés peut impliquer l'action d'hormones telles que l'insuline; toutefois, des travaux complémentaires sont nécessaires pour clarifier leur mode d'action et voir quel peut être le rôle d'autres facteurs endocriniens (IGF 1 et $\mathrm{GH}$, hormones thyroïdiennes).

\section{REMERCIEMENTS}

Nous tenons à remercier la Société Sopharga qui nous a procuré le mélange peptidique utilisé dans cette étude.

\section{RÉFÉRENCES}

Attaix D, Manghebati A, Grizard J, Arnal M (1986) Assessment of in vivo protein synthesis in lamb tissues with $\left[{ }^{3} \mathrm{H}\right]$ valine flooding doses. Biochem Biophys Acta 882, 389-397

Dalle M, Delost $P$ (1976) Plasma and adrenal cortisol concentrations in fotal, newborn and mother guinea-pig during the perinatal period. J Endocrinol 70, 207-214

Garlick PJ, Millward DJ, James WPT (1973) The diurnal response of muscle and liver protein synthesis in vivo in meal-fed rats. Biochem $J$ 136, 935-945

Garlick PJ, Grant I (1988) Amino acid infusion increases the sensitivity of muscle protein synthesis in vivo to insulin. Biochem $J 254$, 579-584

Gray GM, Cooper HL (1971) Protein digestion and absorption. Gastroenterology 61, 535544

Harper AE, Benjamin E (1984) Relationship between intake and rate of oxidation of leucine and $\alpha$-ketoisocaproate in vivo in the rat. $J$ Nutr 114, 431-440

Horn J, Stern MDR, Young $M$, Noakes DE (1983) Influence of insulin and substrate concentration on protein synthetic rate in fetal tissues. Res Vet Sci 35, 35-41

Jepson MM, Bates PC, Millward DJ (1988) The role of insulin and thyroid hormones in the regulation of muscle growth and protein turnover in response to dietary protein in the rat. Br J Nutr 59, 397-415

Matthews DM (1983) Protein digestion and absorption. In: Nutritional Adaptation of the Gastrointestinal tract of the Newborn (Kretchmer N, Minkowski A, eds), Nestlé, Vevey, Raven Press, New York 
Meguid MM, Matthews DE, Bier DM, Meredith CN, Soeldner JS, Young VR (1986) Leucine kinetics at graded leucine intakes in young men. Am J Clin Nutr 43, 781-786

Millward DJ, Bates PC, de Benoist B, Brown JG, Cox M, Halliday D, Odedra B, Rennie MJ (1983) Protein turnover: the nature of the phenomenon and its physiological regulation. V|e Symp int Métabolisme et Nutrition azotés, 1, 69-96, INRA, Versailles

Nissen S, Ostaszewski P (1985) Effects of supplemental dietary energy on leucine metabolism in sheep. Br J Nutr 54, 705-712

Obled C (1987) Aspect dynamique du métabolisme protéique au cours du développement du rat après le sevrage. Contribution à l'évaluation des méthodes de mesure des vitesses d'utilisation métabolique des acides aminés. Thèse de Doctorat d'Etat. Université de Clermont-Ferrand II

Patureau Mirand P, Bernard O, Prugnaud J, Arnal $M$ (1985a) Métabolisme protéique de l'agneau nouveau-né. 2. Influence de l'alimentation sur les flux et le taux de renouvellement des protéines. Reprod Nutr Dév 25, 1061-1073

Patureau Mirand P, Bernard O, Prugnaud J, Levieux D, Pion R (1985b) Métabolisme protéique de l'agneau nouveau-né. 1. Conséquences de l'ingestion de colostrum sur la glycémie, la gammaglobulinémie et les teneurs en acides aminés libres des tissus. Reprod Nutr Dev 25, 1047-1060
Patureau Mirand $P$, Debras $E$, Prugnaud $J$ (1986) Influence de l'ingestion de lactose sur le métabolisme protéique de l'agneau nouveau-né. Reprod Nutr Dév 26, 677-682

Philipps AF, Dubin JW, Raye JR (1981) Maturation of early-phase insuline release in the neonatal lamb. Biol Neonate 39, 225-231

Preedy VR, Garlick PJ (1986) The response of muscle protein synthesis to nutrient intake in post absorptive rats: the role of insulin and amino acids. Biosci Rep 6, 177-183

Reeds PJ, Fuller MF, Cadenhead A, Hay SM (1987) Urea synthesis and leucine turnover in growing pigs: changes during $2 \mathrm{~d}$ following the addition of carbohydrate or fat to the diet. Br J Nutr 58, 301-311

Rérat A, Simoes Nunes $C$, Mendy F, Roger $L$ (1988) Amino acid absorption and production of pancratic hormones in non anaesthetized pigs after duodenal infusions of a milk enzymic hydrolysate or of free amino acids. $\mathrm{Br}$ J Nutr 60, 121-136

Silk DBA, Grimble GK, Rees RG (1985) Protein digestion and amino acid and peptide absorption. Proc Nutr Soc 44, 63-72

Snedecor GW, Cochran WG (1971) Méthodes statistiques, ACTA, Paris, $649 p$

Waterlow JC, Garlick PJ, Millward DJ (1978) Protein turnover in mammalian tissues and in the whole body. North Holland, Amsterdam, $804 \mathrm{p}$ 